\title{
INFLUENCE OF EVEN NUCLEON NUMBERS AND CLOSED SHELLS ON MASS AND CHARGE DISTRIBUTION IN LOW ENERGY FISSION
}

\author{
Z. BUYUKMUMCU,* H. N. ERTEN,** N. K. ARAS* \\ *Middle East Technical University, Ankara (Turkey) \\ **Bilkent University, Ankara (Turkey)
}

(Received November 11, 1993)

\begin{abstract}
Extensive experimental and evaluated yield data are now available for the fragments in low energy nuclear fission. Using these data we re-examined the influence of even nuclear numbers and closed shells on the mass and charge distribution in low energy fission. We used WAHL's Zp model and Ap model. We also examined the effects of $Z=50, N=82$ and possibly $N=88$ shells. A new method was developed based on two Gaussian curves for the even and odd products. The $E O Z$ and $E O N$ values calculated are based on these different methods and are seen to be consistent with each other in spite of the somewhat different definitions of the even-odd effect. The even-odd effect decreases with an increasing fissility parameter. The $E O N$ values are substantially lower than the corresponding $E O Z$ values, probably due to the effect of washing out the neutron pairing effect by prompt neutron emission. The magnitude of the even-odd effect varies with different mass regions. The EOZ and $E O N$ values decrease as they go from asymmetric to symmetric regions in mass distributions.
\end{abstract}

In many studies of nuclear fission, fine structure in mass and charge distribution of the products has been observed. This has been attributed to pairing and shell effects. Increased stability of products with even nucleon numbers and closed shells lead to the enhancement of their yields.

The first quantitative and systematic analysis of the even-odd proton and neutron pairing effects was reported by AMIEL and FELDSTEIN. ${ }^{1}$ WAHL $^{2}$ proposed the Zp Model of charge distribution. The model is based on the assumption of a Gaussian yield distribution modulated by even-odd factors. A different approach was proposed by TRACY et al. ${ }^{3}$ In their method, called the "method of third differences", no knowledge of distribution parameters is necessary. There are also several model independent calculations. CLERC et al. ${ }^{4}$ introduced the concept of global even-odd effects in fission.

Closed shells affect the fission process in two ways. First it is widely believed that mass asymmetry is caused by the spherical shells, of $Z=50$ and $N=82$ and the deformed shell $N=88$. Second, the yields of closed shell nuclides are enhanced leading to fine structure in the mass distribution. ${ }^{5}$ NAKAHARA et al. ${ }^{6}$ used two Gaussian functions, one centered at $N=82$ and the other at $N=88$ to describe experimental mass distributions. The enhancement of nuclide yields is thus due to both closed shells as well as nucleon pairing. 
IZAK-BIRAN and $\mathrm{AMIEL}^{7}$ estimated the magnitude of shell effects in the fast neutron induced fission of ${ }^{232} \mathrm{Th}$. In another study by NAEUMANN et al. ${ }^{8}$ no pronounced effects of the doubly magic ${ }^{132} \mathrm{Sn}$ on charge dispersion was found. Neutron shell effects were studied in detail by TOKAY and TALAT-ERBEN 9 and TOKAY. ${ }^{10}$ Their calculations were based on the $A_{p}$ model. The variances of the left and right sides of the Gaussian yield curves were found to be significantly different. This discontinuity was interpreted as resulting from the spherical shell at $N=82$ and the deformed shell at $N=88$. Recently GÖNNENWEIN ${ }^{11}$ surveyed various approaches to assess odd-even effects in fission yields.

Extensive experimental and evaluated yield data are now conveniently available on tapes and discs. We wantd to re-examine the fine structure effects in low energy fission and extend the studies beyond thermal neutron fission of ${ }^{233} \mathrm{U},{ }^{235} \mathrm{U}$ and ${ }^{239} \mathrm{Pu}$. Starting with WAHL's $Z_{p}$ and $A_{p}$ models, ${ }^{12}$ methods were developed to estimate the magnitude of even-odd proton and neutron pairing as well as shell effects. Contributions of both pairing and shell structure were investigated. Even-odd factors obtained from different models are compared and their variations as a function of the fissility parameter, $Z^{2} / A$, is examined.

\section{Calculations based on the $Z_{p}$ model}

Method of calculation: In the $Z_{p^{i}}$ model, the nuclear charge distribution within a mass chain is represented by a Gaussian function. ${ }^{12,13}$ The maximum point is the most probable charge. $Z_{p}$. The smooth curve is distorted by the even number of neutrons and/or protons. The modulated Gaussian function of fractional independent yields is given as follows;

$$
F I(Z)=[N F][E O F] \int_{Z \rightarrow 0.5}^{Z+0.5} P(Z) \mathrm{d} Z
$$

where $F I(Z)$ - the fraction independent yield of element $Z$,

$N F$ - normalization factor,

$P(Z)$ - probability function defined as

$$
P(Z)=\frac{1}{\sqrt{\pi c}} \exp \left[-\frac{\left(Z-Z_{p}\right)^{2}}{c}\right]
$$

where $c$ is the width parameter, related to charge dispersion $\sigma$ through Sheppards correction $c=2\left(\sigma^{2}+(1 / 12)\right)$. 
EOF is the even-odd factor defined as EOZ.EON, EOZIEON, EON/EOZ and $1 /(E O Z \cdot E O N)$ for even-even, even-odd, odd-even and odd-odd products, respectively. 2,12,13,14 Here $E O Z$ and $E O N$ are even-odd enhancement factors for proton and neutron, respectively.

The original least-squares program used by WAHL ${ }^{15}$ was adapted to the $\mathrm{HB}-6 / 80$ at MAINZ by MEIXLER. ${ }^{16}$ This version was adapted to our IBM 3090 computer system and used in our calculations.

Results and discussion: The experimental yield data compiled by $\mathrm{WAHL}^{12}$ and by $\mathrm{CHUNG}^{17}$ were used in the calculations. The three parameters, $Z_{p}, \sigma$, and $E O F$ were calculated for those mass chains with sufficient yield data in the thermal neutron induced fission of ${ }^{233} \mathrm{U},{ }^{235} \mathrm{U},{ }^{239} \mathrm{Pu}$ and the spontaneous fission of ${ }^{252} \mathrm{Cf}$. The results are summarized in Table 1.

The values of the Gaussian width parameter, $\sigma$, for different mass chains, fluctuate around the value of $0.6 \pm 0.2$. It is however clear that it is not possible to define all masss chains with one single $\sigma$ value. The decrease in $\sigma$ for $A=138$ mass chain may be attributed to the $N=88$.

The third charge distribution parameter, the: even-odd factor, $E O F$, varies systematically between even and odd $A$ mass chains. The EOF factors are generally higher for even $A_{!}$chains compared to neighboring odd ones. The $E O F$ factors are seen to depend on the nature of the fissioning nuclide. For spontaneous fission of ${ }^{252} \mathrm{Cf}$ no significant $E O F$ factors were observed. Average charge dispersion parameters (global parameters) can be obtained for each fissioning nuclide when all available yield data are used in the Gaussian fits. ${ }^{12,17,18,19}$ The average parameters determined using all available yield data for several fissioning nuclides are listed in Table 2. The charge polarization function, $\triangle Z$, which is defined as $Z_{p}-Z_{U C D}$, where $Z_{U C D}$ is the charge calculated assuming unchanged charge density of compound nuclei. $\Delta Z$ of individual mass chains can be calculated using $\mathscr{L}(A=140)$ as given by WAHL. ${ }^{12}$ It is interesting that ${ }^{252} \mathrm{Cf}$ with the highest fissility parameter has the lowest polarization. The intrinsic excitation energy increases with increasing fissility parameter. This seems to prevent further polarization. Increasing excitation energy of a nucleus thus decreases its polarization.

A systematic increase is observed in the Gaussian width parameters $\sigma$ with increasing mass number of the fissioning nucleus. As stated before, the mass change, $\delta A / \delta Z$ increases with increasing $A$, resulting in a lower charge density, $\delta Z / \delta A$. This leads to an increase in isobaric products and to a broadening of the charge distribution curve.

The even-odd factors were calculated for different mass regions in ${ }^{235} \mathrm{U}$ fission, where enough yield data were available. The highest values of EOF's were observed in the very asymmetric regions. The even-odd effect seems to disappear in the symmetric region. From binding energy considerations the highest energy is released in the 
Z. BUYUKMUMCU et al.: INFLUENCE OF EVEN NUCLEON NUMBERS




Table 2

$Z_{p}$ model global fit parameters for various fissioning nuclides

\begin{tabular}{lcccccc}
\hline $\begin{array}{l}\text { Fissioning } \\
\text { nuclides* }\end{array}$ & $\begin{array}{c}\text { Yield data } \\
\text { from Ref. }\end{array}$ & $Z^{2} / A$ & $E O Z$ & $E O N$ & $\sigma$ & $\Delta Z(140)$ \\
\hline $\begin{array}{l}{ }^{232} \mathrm{Th} \\
{ }^{235} \mathrm{U}\end{array}$ & 19 & 34.76 & 1.222 & 1.187 & 0.557 & 0.529 \\
$\begin{array}{l}\text { (all } A) \\
235\end{array}$ & 12 & 35.86 & 1.305 & 1.052 & 0.493 & 0.451 \\
$108-126$ & 12 & 35.86 & 0.949 & 0.806 & 0.699 & 0.897 \\
${ }^{235} \mathrm{U}$ & 12 & 35.86 & 1.348 & 1.040 & 0.525 & 0.933 \\
$\begin{array}{l}A>145 \\
{ }^{235} \mathrm{U}\end{array}$ & 12 & 35.86 & 1.340 & 1.273 & 0.549 & 0.997 \\
$A<82$ & 12 & 36.17 & 1.201 & 0.998 & 0.480 & 0.489 \\
${ }^{233} \mathrm{U}$ & 19 & 36.51 & 0.997 & 1.021 & 0.538 & 0.436 \\
${ }^{241} \mathrm{Pu}$ & 12 & 36.82 & 1.081 & 1.025 & 0.571 & 0.502 \\
${ }^{239} \mathrm{Pu}$ & 12 & 38.11 & 1.044 & 0.926 & 0.613 & 0.393 \\
${ }^{252} \mathrm{Cf}$ & 12 & & & & & \\
\hline
\end{tabular}

*All the fissions are with thermal neutrons except ${ }^{232} \mathrm{Th}$ (fast neutrons) and ${ }^{252} \mathrm{Cf}$ (spontaneous fission).

symmetric region and the lowest in the very asymmetric region. Thus the even-odd effect is expected to decrease as the energy available for pair breaking (released energy) increases.

When the experimental yields, of Rudstam et al. ${ }^{18}$ for ${ }^{238} \mathrm{U}$ fission were used in the calculations, no satisfactory fit to the $Z_{p}$-model was possible. A selected set of data using only fractional independent yields, which were appreciably greater than zero, gave results similar to those obtained by using WAHL's yield data. ${ }^{12}$

\section{Calculations based on the Ap model}

Method of calculation: In the $A_{p}$ model, the distribution of the independent yields of all isotopes at constant $Z$, is described by a Gaussian function. ${ }^{12} A^{\prime}$ values, the mass of primary fragments, must be used. It is not possible to know the exact number of neutrons emitted by each individual fission product.

However, average number of neutrons emitted per fragment, $\bar{v}$, can be calculated by using TERREL's method. ${ }^{20} A^{\prime}$ values were then determined from the relation 
$A^{\prime}=A+\bar{v}$. The modified Gaussian function in this treatment is given as follows;

$$
Y(A)=[N F][E O F][Y(Z)] \int_{A-0.5}^{A+0.5} P(A) \mathrm{d} A
$$

where $P(A)$ is the probability function given by

$$
P(A)=\frac{1}{\sqrt{2 \pi \sigma^{2}}} \exp \left[-\frac{\left(A-A_{p}\right)^{2}}{2 \sigma^{2}}\right]
$$

$Y(A)$ is the independent isotopic yield of isotope $A, A_{p}$ is the most probable mass of an isotopic mass dispersion curve, $N F$ is the normalization factor and $Y(Z)$ is the total independent isotopic yield.

Table 3

Results of $A_{p}$ model calculations based on experimental yields for the thermal neutron fission

\begin{tabular}{|c|c|c|c|c|c|c|c|c|c|}
\hline \multirow{2}{*}{$Z$} & \multicolumn{3}{|c|}{$A_{p}$} & \multicolumn{3}{|c|}{$\sigma$} & \multicolumn{3}{|c|}{$E O N$} \\
\hline & ${ }^{233} \mathrm{U}$ & ${ }^{235} \mathrm{U}$ & ${ }^{239} \mathrm{p}_{\mathrm{u}}$ & ${ }^{23} \mathrm{U}$ & ${ }^{235} \mathrm{U}$ & ${ }^{239} \mathrm{Pu}$ & ${ }^{233} \mathrm{U}$ & ${ }^{235} \mathrm{U}$ & ${ }^{239} \mathrm{Pu}$ \\
\hline 31 & & 80.436 & & & 1.679 & & & 1.350 & \\
\hline 32 & & 81.869 & & & 1.285 & & & 1.152 & \\
\hline 33 & 83.459 & 84.222 & & 1.401 & 1.417 & & 1.062 & 1.080 & \\
\hline 34 & 85.519 & 86.635 & & 1.324 & 1.474 & & 1.046 & 1.155 & \\
\hline 35 & 87.966 & 88.813 & 87.269 & 1.338 & 1.314 & 2.228 & 1.033 & 1.065 & 0.988 \\
\hline 36 & 91.034 & 91.034 & 90.824 & 1.363 & 1.363 & 1.293 & 1.063 & 1.063 & 1.233 \\
\hline 37 & 92.631 & 93.607 & 93.276 & 1.369 & 1.412 & 1.463 & 1.032 & 1.036 & 0.989 \\
\hline 38 & 95.183 & 96.096 & 95.513 & 1.552 & 1.599 & 1.473 & 1.022 & 1.026 & 1.017 \\
\hline 39 & 97.523 & 98.748 & 98.070 & 1.510 & 1.592 & 1.472 & 1.089 & 1.049 & 1.083 \\
\hline 40 & 100.370 & 101.150 & 100.720 & 1.641 & 1,501 & 1.528 & 1.043 & 1.134 & 1.144 \\
\hline 41 & 102.440 & 102.990 & 102.990 & 1.470 & 1.311 & 1.464 & 1.181 & 1.228 & 1.115 \\
\hline 42 & & & 105.830 & & & 1.180 & & & 1.045 \\
\hline 43 & & & 107.290 & & & 1.136 & & & 1.284 \\
\hline 44 & & & 132.100 & & & 1.807 & & & 1.642 \\
\hline 50 & & 130.670 & & & 1.481 & & & 1.068 & \\
\hline 51 & 132.230 & 132.630 & 133.340 & 1.875 & 1.486 & 1.812 & 1.290 & 1.172 & 1.015 \\
\hline 52 & 134.590 & 135.670 & & 1.530 & 1.491 & & 0.766 & 1.002 & \\
\hline 53 & 136.460 & 137.330 & 136.940 & 1.540 & 1.491 & 1.656 & 0.994 & 1.162 & 0.992 \\
\hline 54 & 138.020 & 139.320 & 139.430 & 1.714 & 1.557 & 1.382 & 1.130 & 1.111 & 0.995 \\
\hline 55 & & 142.220 & & & 1.427 & & & 0.877 & \\
\hline 56 & & 143.820 & & & 1.996 & & & 1.157 & \\
\hline
\end{tabular}
of ${ }^{233} \mathrm{U},{ }^{235} \mathrm{U},{ }^{239} \mathrm{Pu}$ 
Since the proton number is constant, only the even-odd neutron effect can be determined in this treatment. $E O F$ is equal to $E O N$ for even neutrons and to $1 / E O N$ for odd neutrons. The least-squares program was modified for $A_{p}$ calculations.

Results and discussion: In the $A_{p}$ model, the isotopic mass dispersion curve has three parameters; $A_{p}, \sigma$, and $E O N$. Since it was not possible to assign even or odd neutron numbers to products from the noninteger $A^{\prime}$ values, the $A$ values were used for the assignments.

There are substantially more, as many as 10 times, yield data for isotopic products of a given element, than the isobaric products of the $Z_{p}$ model. This is because isotopic chains are longer than isobaric chains. The results of $A_{p}$ model calculations for ${ }^{233} \mathrm{U}$, ${ }^{235} \mathrm{U}$ and ${ }^{239} \mathrm{Pu}$ fission are summarized in Table 3. The width $\sigma$, of the isotopic mass dispersion curves fluctuates around 1.50 for the three fissioning nuclides as compared to 0.65 in the $Z_{p}$ model.

In the $Z=31$ isotopic chain, a high EON value of 1.35 was observed. This chain includes products with the $N=50$ shell, which may contribute to the $E O N$ factor. Similarly, the $Z=51$ isotopic chain may have contributions from the $N=82$ neutron shell.

Generally the magnitudes of $E O N$ are found to be consistent with those from the $Z_{p}$ model.

\section{Calculation of the shell effect}

Calculation method: Both isotopic and isotonic independent yield distributions in low energy fission show Gaussian behavior. Experimental yields fluctuate around the Gaussian curve due to both even-odd nucleons and closed shells. It is, however, difficult to separate the two contributions. In order to neutralize the pairing effect, only products with even protons or neutrons were considered. Since low yields are generally associated with large errors, yields which were less than half the most probable yield were discarded. A nonlinear regression program was written to evaluate the Gaussian parameters and the magnitude of the shell effect.

The independent yield of even $N$ isotones is defined by the function;

$$
I Y(N)=\frac{1}{\sqrt{2 \pi \sigma^{2}}} \exp \left[-\frac{\left(N-N_{p}\right)^{2}}{2 \sigma^{2}}\right]
$$

Here, $N_{p}$ is the most probable $N$ for the isotonic dispersion curve. The distribution curve obtained for the product in ${ }^{235} \mathrm{U}$ fission is shown in Fig. 1. It is clearly seen that the yields of spherical and deformed shell nuclides with $N=82$ and $N=88$, 


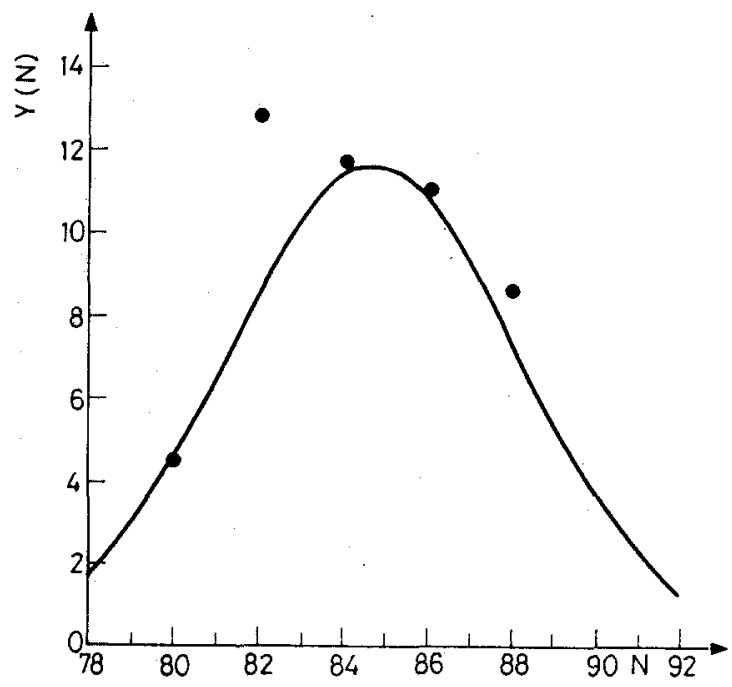

Fig. 1. Isotonic Gaussian yield curve obtained using experimental even $N$ yields in ${ }^{235} \mathrm{U}$ fission. $\bullet$ experimental value, - fitted curve

Table 4

Isotopic yield ratios of fission products around the $Z=50$ shell

\begin{tabular}{cccc}
\hline Fissioning nuclide & $Y(48) / Y(47)$ & $Y(50) / Y(49)$ & $Y(52) / Y(51)$ \\
\hline${ }^{232} \mathrm{Th}$ & 0.940 & 29.599 & 2.260 \\
${ }^{233} \mathrm{U}$ & 1.850 & 20.221 & 2.456 \\
${ }^{235} \mathrm{U}$ & 1.900 & 10.893 & 2.614 \\
${ }^{239} \mathrm{Pu}$ & 0.551 & 8.550 & 2.174 \\
${ }^{24} 1_{\mathrm{Pu}} \mathrm{Pu}$ & 1.069 & 6.082 & 1.908 \\
${ }^{243} \mathrm{Cm}$ & 0.285 & 8.036 & 1.732 \\
$245 \mathrm{Cm}$ & 0.320 & 12.375 & 1.461 \\
$252 \mathrm{Cf}$ & 0.381 & 7.966 & 1.716 \\
\hline
\end{tabular}

respectively, are enhanced. Using the deviations from the Gaussian curve, a quantitative estimate of the shell effect may be made.

For $N=50$ and $Z=50$ species the independent yields were too low to allow such a treatment.

Results and discussion: An important shell effect in fission is the asymmetric distribution of mass. The asymmetry is attributed to $Z=50, N=82$, and possibly $N=88$ shells.

The isotopic yreld ratios of fission products near $Z=50$ for several fissioning nuclides are given in Table 4. It is seen that the yield ratio $Y(50) / Y(49)$ is strikingly 
higher than the neighboring pairs in all fissions. Besides the fast changing mass distribution in that region, this may be taken as an indication of yield enhancement by the $Z=50$ shell.

As stated earlier by using even $N$ products only, a quantitative estimate of the shell effect is possible. The results of such a calculation for several fissioning nuclides are

Table 5

Magnitude of the shell effect on the isotonic yield distributions of several fissioning systems

\begin{tabular}{ccc}
\hline $\begin{array}{c}\text { Fissioning } \\
\text { nuclide }\end{array}$ & $\begin{array}{c}\text { Shell effect, } \% \\
(N=82)\end{array}$ & $\begin{array}{c}\text { Shell effect, } \% \\
(N=88)\end{array}$ \\
\hline${ }^{233} \mathrm{U}$ & 20.5 & 17.6 \\
$235 \mathrm{U}$ & 48.6 & 16.7 \\
$239 \mathrm{Pu}$ & 33.7 & 17.8 \\
$241 \mathrm{Pu}$ & 50.3 & -4.6 \\
$243 \mathrm{Cm}$ & 5.8 & 24.6 \\
$252 \mathrm{Cf}$ & 12.7 & 8.4 \\
\hline
\end{tabular}

given in Table 5. The shell effects are given as percentage enhancements relative to normal Gaussian yields. The $N=82$ spherical shell effect appears to be more pronounced than that of the $N=88$ deformed shell.

\section{Calculations based on the two Gaussian method}

Calculation method: In the isotonic and isotopic yield distributions, the even and odd products may be assumed to follow two different Gaussian curves. In this method two different parameter sets are calculated for the even and the odd products separately, using the following relations;

$$
\begin{aligned}
& Y_{e}(Z)=\frac{1}{\sqrt{2 \pi \sigma_{e}^{2}}} \exp \left[-\frac{\left(Z-Z_{p}\right)^{2}}{2 \sigma_{e}^{2}}\right] \\
& Y_{o}(Z)=\frac{1}{\sqrt{2 \pi \sigma_{o}^{2}}} \exp \left[-\frac{\left(Z-Z_{p}\right)^{2}}{2 \sigma_{o}^{2}}\right]
\end{aligned}
$$

The most probable charge, $Z_{p}$, of the two curves are assumed to be equal. The even curve is assumed to be enhanced by a factor of $E O Z$ and the odd curve suppressed by a 
factor of $1 / E O Z$ relative to the normal Gaussian curve. The yield ratio at the most probable charge $Z_{p}$ can be shown to be;

$$
\frac{Y_{e}\left(Z_{p}\right)}{Y_{o}\left(Z_{p}\right)}=\frac{Y\left(Z_{p}\right)^{*} E O Z}{Y\left(Z_{p}\right)^{*} \frac{1}{E O Z}}
$$

where $Y\left(Z_{p}\right)$ is the normal yield at $Z_{p}$. Since $Z-Z_{p}=0$ at $Z=Z_{p}$, from Eq.s (6)-(8);

$$
\begin{array}{r}
{[E O Z]^{2}=\frac{\frac{1}{\sigma_{e} \sqrt{2 \pi}} e^{o}}{\frac{1}{\sigma_{o} \sqrt{2 \pi}} e^{o}}} \\
E O Z=\sqrt{\frac{\sigma_{o}}{\sigma_{e}}}
\end{array}
$$

where $\quad \sigma_{e}-$ width of the Gaussian curve of the even $Z$ products,

$\sigma_{o}$ - width of the Gaussian curve of the odd $Z$ products.

A similar expression is obtained for $E O N$ in the isotonic yield distribution.

Results and discussion: The even and odd isotopic yield distribution curves in ${ }^{235} \mathrm{U}$ fission are shown in Fig. 2. The $\sigma$ values of the even and odd yield curves in ${ }^{233} \mathrm{U},{ }^{235} \mathrm{U}$, ${ }^{239} \mathrm{Pu}$ and ${ }^{252} \mathrm{Cf}$ fission are given in Table 6 . The corresponding EOZ and EON values calculated according to Eq. (10) are also given. The results are in good agreement with those from other methods.

Table 6

\begin{tabular}{|c|c|c|c|c|c|c|c|c|c|c|c|c|}
\hline \multirow{2}{*}{$\begin{array}{l}\text { Fission- } \\
\text { ing } \\
\text { nuclide }\end{array}$} & \multicolumn{3}{|c|}{ Light $Z$} & \multicolumn{3}{|c|}{ Light $N$} & \multicolumn{3}{|c|}{ Heavy $Z$} & \multicolumn{3}{|c|}{ Heavy $N$} \\
\hline & $\sigma_{e}$ & $\sigma_{o}$ & $E O Z$ & $\sigma_{e}$ & $\sigma_{o}$ & $E O N$ & $\sigma_{e}$ & $\sigma_{o}$ & $E O Z$ & $\sigma_{e}$ & $\sigma_{o}$ & $E O N$ \\
\hline${ }^{235} \mathrm{U}$ & 2.1 & 3.0 & 1.20 & 3.4 & 3.8 & 1.06 & 2.1 & 3.1 & 1.21 & 3.2 & 3.7 & 1.08 \\
\hline${ }^{233} \mathrm{U}$ & 2.1 & 3.0 & 1.20 & 3.3 & 3.7 & 1.06 & 2.1 & 2.9 & 1.18 & 3.3 & 3.7 & 1.06 \\
\hline${ }^{239} \mathrm{Pu}$ & 2.2 & 2.6 & 1.09 & 3.5 & 3.8 & 1.04 & 2.2 & 2.6 & 1.09 & 3.2 & 3.5 & 1.03 \\
\hline${ }^{252} \mathrm{Cf}$ & 2.6 & 2.6 & 1.00 & 3.6 & 3.7 & 1.01 & 2.6 & 2.6 & 1.00 & 3.8 & 3.7 & 0.99 \\
\hline
\end{tabular}

Even and odd sigma values of isotopic and isotonic yield curves and the corresponding $E O Z$ and $E O N$ values for ${ }^{238} \mathrm{U},{ }^{235} \mathrm{U},{ }^{239} \mathrm{Pu}$ and ${ }^{252} \mathrm{Cf}$ fission 


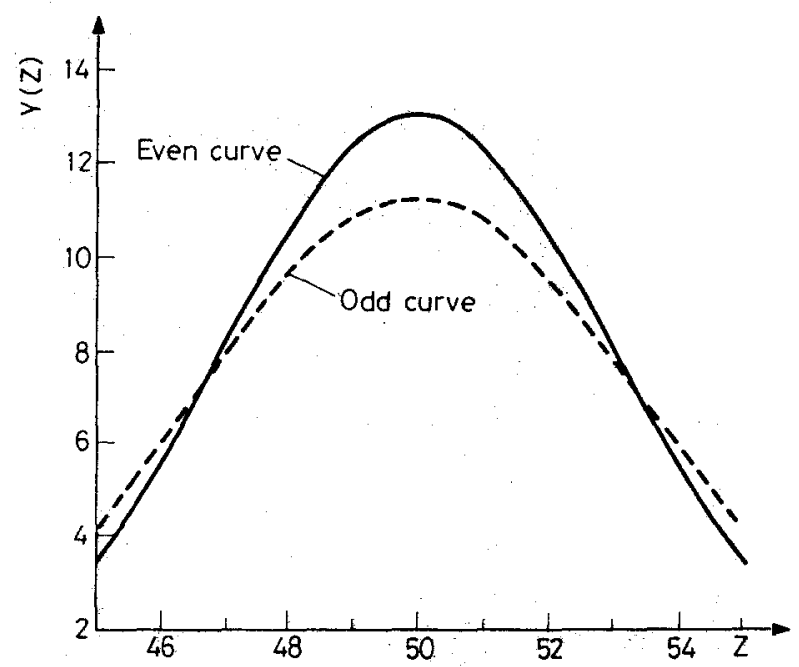

Fig. 2. Even and odd isotopic yield distribution curves in ${ }^{235} U$ fission used in the two Gaussian method

\section{Model independent calculations}

There is a direct method as discussed by ERTEN and ARAS ${ }^{21}$ to determine the magnitude of the even-odd effect in fission. Here $E O Z$ is defined as

$$
E O Z=\frac{2 \sum_{\text {all }}^{Z} Y_{e}(Z)}{2 \sum_{\text {all }}^{Z} Y_{e}(Z)+2 \sum_{\text {all }}^{Z} Y_{o}(Z)}
$$

where $E O Z$ - even-odd proton enhancement factor,

$Y_{e}(Z)$ - isotopic independent yield of even $Z$ products,

$Y_{o}(Z)$ - isotopic independent yield of odd $Z$ products.

A similar expression was used for evaluating the even-odd neutron enhancement factor EON. Experimental as well as compiled and evaluated yield data were used. ${ }^{12,17,19}$. The results are given in Table 7 . The results are consistent with previous calculations. 
Table 7

Model independent results using WAHL's data

\begin{tabular}{lrrrc}
\hline & ${ }^{233} \mathrm{U}$ & \multicolumn{1}{c}{${ }^{235_{\mathrm{U}}}$} & ${ }^{239} \mathrm{Pu}$ & ${ }^{252} \mathrm{Cf}$ \\
\hline$\Sigma_{o} Y(Z)$ & 59.240 & 73.915 & 69.985 & 28.051 \\
$\Sigma_{e} Y(Z)$ & 89.374 & 117.280 & 86.044 & 48.998 \\
$\Sigma_{o} Y(N)$ & 73.142 & 90.441 & 75.450 & 26.535 \\
$\Sigma_{e} Y(N)$ & 75.472 & 100.759 & 80.580 & 50.514 \\
$E O Z$ & 1.203 & 1.227 & 1.103 & - \\
$E O N$ & 1.016 & 1.054 & 1.038 & $\Sigma_{o} Y(Z)$
\end{tabular}

Number of nuclides

$\begin{array}{lrrrr}\text { Even } Z & 73 & 82 & 70 & 33 \\ \text { Odd } Z & 75 & 106 & 73 & 17 \\ \text { Even } N & 69 & 88 & 66 & 32 \\ \text { Odd } N & 79 & 100 & 77 & 18 \\ \text { Even-Even } & 34 & 38 & 33 & 27 \\ \text { Even-Odd } & 39 & 44 & 37 & 6 \\ \text { Odd-Even } & 35 & 50 & 33 & 5 \\ \text { Odd-Odd } & 40 & 56 & 40 & 12\end{array}$

Specific yields

\begin{tabular}{lllll} 
Even $Z$ & 1.224 & 1.430 & 1.229 & - \\
Odd $Z$ & 0.780 & 0.697 & 0.959 & - \\
Even $N$ & 1.074 & 1.145 & 1.221 & - \\
Odd $N$ & 0.926 & 0.904 & 0.980 & - \\
Even-Even & 1.272 & 1.603 & 1.239 & - \\
Even-Odd & 1.183 & 1.282 & 1.227 & - \\
Odd-Even & 0.921 & 0.797 & 1.283 & - \\
Odd-Odd & 0.675 & 0.608 & 0.757 & - \\
\hline
\end{tabular}

\section{Conclusion}

Table 8 summarizes $E O N$ and $E O Z$ values determined by different methods considered in this work. The results are seen to be consistent with each other despite the somewhat different definitions of the even-odd effect.

The even-odd effect decreases with increasing fissility parameter. This in turn is related to the increase in intrinsic excitation energy. ${ }^{5}$

The $E O N$ vaiues are lower than the corresponding $E O Z$ values probably due to prompt neutron emission. 
Table 8

Summary of $E O Z$ and $E O N$ results obtained by various methods in this work

\begin{tabular}{ccccccccc}
\hline & & \multicolumn{3}{c}{ EOZ } & & EON & \\
\cline { 3 - 8 } $\begin{array}{c}\text { Fissioning } \\
\text { nuclides }\end{array}$ & $Z^{2} / A$ & $\begin{array}{c}Z_{p} \\
\text { model }\end{array}$ & $\begin{array}{c}\text { Model } \\
\text { inde- } \\
\text { pendent }\end{array}$ & $\begin{array}{c}\text { Two } \\
\text { Gaussian } \\
\text { model }\end{array}$ & $\begin{array}{c}Z_{p} \\
\text { model }\end{array}$ & $\begin{array}{c}\text { Model } \\
\text { inde- } \\
\text { pendent }\end{array}$ & $\begin{array}{c}\text { Two } \\
\text { Gaussian } \\
\text { model }\end{array}$ \\
\hline${ }^{232} \mathrm{Th}$ & 34.76 & 1.22 & 1.21 & - & 1.19 & 1.01 & - \\
${ }^{235} \mathrm{U}$ & 35.86 & 1.30 & 1.24 & 1.21 & 1.05 & 1.07 & 1.07 \\
${ }^{233} \mathrm{U}$ & 36.17 & 1.20 & 1.23 & 1.20 & 1.00 & 1.07 & 1.06 \\
${ }^{241} \mathrm{Pu}$ & 36.51 & 1.00 & 1.14 & - & 1.02 & 1.05 & - \\
${ }^{239} \mathrm{Pu}$ & 36.82 & 1.08 & 1.14 & 1.09 & 1.03 & 1.05 & 1.04 \\
${ }^{245} \mathrm{Cm}$ & 37.46 & 1.07 & 1.03 & - & 0.96 & 1.03 & - \\
${ }^{243} \mathrm{Cm}$ & 37.77 & - & 1.03 & - & - & 1.03 & - \\
${ }^{252} \mathrm{Cf}$ & 38.11 & 1.04 & 1.00 & 1.00 & 0.93 & 1.01 & 1.00 \\
\hline
\end{tabular}

The magnitude of the even-odd effect varies for different mass regions. The $E O Z$ and $E O N$ values decrease on going from the asymmetric to the symmetric region of the mass distribution.

This paper is dedicated to Ridvan K. TOKAY, late Professor of Chemistry, recognized authority in nuclear and physical chemistry. We all miss his insights, creativity and wit.

\section{References}

1. S. AMIEL, H. FELDSTEIN, in: Proc. 3rd Int. Symp. on Physics and Chemistry of Fission, Rochester, 1973, paper No. IAEA/SM-174/25, 1973.

2. A. C. WAHL, J. Radioanal. Chem., 55 (1980) 111.

3. B. L. TRACY, J. CHAUMONT, R. KLAPISCH, J. M. NITSCHKE, A. M. POSKANZER, E. ROECKL C. THIBAULT, Phys. Rev., C5 (1972) 222.

4. H. G. CLERC, W. LANG, H. WOHLFORT, K.-H. SCHIMIDT, H. SCHRADER, K. E. PFERDEKAMPFER, R. JUNGMANN, Z. Phys., A274 (1975) 203.

5. B. D. WILKINS, E. B. STEINBERG, R. R. CHASSMAN, Phys. Rev., C14 (1976) 1832.

6. H. NAKAHARA, T. OHTSUKI, Y. HAMAJIMA, K. SUCKI, Radiochim. Acta, 43 (1988) 77.

7. T. IZAK-BIRAN, S. AMIEL, Phys. Rev., C16 (1977) 266.

8. R. NAEUMANN, H. FOLGER, H. O. DENSCHLAG, J. Inorg. Nucl. Chem., 34 (1972) 1785.

9. TOKAY, M. TALAT-ERBEN, Phys. Rev, C19 (1979) 871.

10. R. K. TOKAY, Ph.D. Thesis, Technical University of Istanbul, Department of Physical Chemistry, 1977. 11. F. GÖNNENWEIN, Nucl. Inst. Methods Phys. Res., A316 (1992) 405. 
12. A. C. WAHL, Atomic Data and Nuclear Data Tables, 39 (1988) 1.

13. A. C. WAHL, Nuclear Charge Distribution in Fission, New Directions in Physics, Acad. Press, New York, 1987, p. 163.

14. H.-H. MEIXLER, K. WOLFSBERG, H. O. DENCHLAG, Can. J. Chem. 61 (1983) 665.

15. L. A. BUSING, H. A. LEVY, Oak Ridge National Lab., Repont ORNL-TM-271, 1962.

16. H.-H. MEIXLER, Ph.D. Thesis, Institự für Kernchemie, Unjversitất Mainz, Germany.

17. CHIEN CHUNG, Radiochim. Acta, 39 (1986) 113.

18. G. RUDSTAM, P. AAGARD, B. EKSTROM, E. LUND, H. GOKTURK, H. U. ZWICKY, Radiochim. Acta, 49 (1990) 155.

19. M. F. JAMES, R. W. MLLS, D. R. WEAVER, A New Evaluation of Fission Product Yields and the Production of a New Library (UKFY2) of Independent and Cumulative Yields, Part Il, Tables of Measured and Recommended Fission Yields, AEA-TRS-1018, 1990.

20. J. TERREL, Phys. Revi, 127 (1962) 880.

21. H. N. ERTEN, N. K. ARAS, J. Radioanal. Nucl. Chem., 158 (1992) 41. 